\title{
Small flux superpotential in F-theory compactifications
}

\author{
Yoshinori Honma ${ }^{1, *}$ and Hajime Otsuka ${ }^{2, \dagger}$ \\ ${ }^{1}$ Department of Current Legal Studies, Meiji Gakuin University, Yokohama, Kanagawa 244-8539, Japan \\ ${ }^{2}$ Theory Center, Institute of Particle and Nuclear Studies, High Energy Accelerator Research Organization, \\ KEK, 1-1 Oho, Tsukuba, Ibaraki 305-0801, Japan
}

(Received 12 March 2021; accepted 25 May 2021; published 22 June 2021)

\begin{abstract}
We investigate whether a class of models describing F-theory compactifications admits a specific type of flux vacua with an exponentially small vacuum expectation value of the superpotential, by generalizing a method recently developed in type IIB flux compactifications. First we clarify that a restricted choice of $G_{4}$-flux components reduces a general flux superpotential into a simple form, which promotes the existence of supersymmetric vacua with one flat direction at the perturbative level. Then we utilize the techniques of mirror symmetry to determine one-instanton corrections to the potential and investigate in detail the vacuum solutions of a particular model.
\end{abstract}

DOI: $10.1103 /$ PhysRevD.103.126022

\section{INTRODUCTION}

Stabilization of moduli fields arising from space-time compactifications is of particular relevance for the construction of four-dimensional realistic universe from string theory as well as higher-dimensional gravity. Moduli fields correspond to not only the size and shape of the extra dimension, but also determine various couplings in the resultant four-dimensional effective theory. In order not to be contradicted with the observational constraints obtained from the realm of cosmology and phenomenology, one needs to fix the dynamics of moduli fields adequately.

One of the well-established frameworks of moduli stabilization has been realized in type IIB string theory on Calabi-Yau orientifolds. There the Ramond-Ramond and Neveu-Schwarz fluxes in string theory stabilize the complex structure moduli and the axio-dilaton, while the Kähler moduli can be stabilized by nonperturbative corrections. More precisely, it is quite well known that the imaginary selfdual three-form fluxes in type IIB string theory can provoke stable Minkowski minima, on which complex structure moduli and axio-dilaton are stabilized at a compactification scale [1]. In the Kachru-Kallosh-Linde-Trivedi (KKLT) model [2] and the LARGE Volume Scenario developed in [3], it has been shown that perturbative stringy $\alpha^{\prime}$ corrections and nonperturbative instanton corrections with appropriate

\footnotetext{
*yhonma@law.meijigakuin.ac.jp

'hotsuka@post.kek.jp
}

Published by the American Physical Society under the terms of the Creative Commons Attribution 4.0 International license. Further distribution of this work must maintain attribution to the author(s) and the published article's title, journal citation, and DOI. Funded by SCOAP ${ }^{3}$. uplifting mechanism completely fix the remaining Kähler moduli, providing the outline of the construction of de Sitter space-time in string theory context. There the smallness of the value of flux-induced superpotential for complex structure moduli fields played a crucial role.

Recently, the authors of [4] (see also [5,6]) described a feasible method for constructing flux vacua with exponentially suppressed superpotential in the framework of type IIB Calabi-Yau orientifolds, which can be naturally incorporated into the KKLT construction. The main idea to obtain such a small flux superpotential is to utilize nonperturbative terms in the prepotential to the fullest extent. More precisely, they first neglected nonperturbative corrections in the prepotential and made a restricted choice of background fluxes such that the possible minima at the perturbative level admit a flat direction along with exactly vanishing superpotential. Then, by taking into account the nonperturbative corrections to the effective theory, the remaining modulus acquires a small mass and they found preferable minima with exponentially small flux superpotential. This mechanism is so simple and thus have a broad range of applicability, especially toward explicit constructions of KKLTlike scenario in various effective field theories.

In this paper, we evaluate the validity of the above method in the framework of F-theory flux compactifications, where both the open and closed string moduli fields can be geometrically controlled [7]. Extending the type IIB setup of [4] to F-theory compactified on Calabi-Yau fourfolds, we clarify how to obtain a specific type of perturbatively flat vacua with exactly vanishing superpotential. After specifying an explicit example for the background, we utilize the mirror symmetry of Calabi-Yau fourfolds to compute relevant nonperturbative corrections to the potential. To solve intricate equations of the system 
appropriately, we rely on a numerical calculation and investigate whether a desirable class of F-theory vacua with small superpotential can be allowed in our setup. Our verification may shed new light on a realization of KKLTlike scenario over a broad range of stringy frameworks.

This paper is organized as follows. First we briefly look at a general formula about the effective theory arising from F-theory compactifications in Sec. II. Then we pick up a particular Calabi-Yau fourfold as an explicit example, and numerically investigate the model to verify the existence of flux vacua equipped with the exponentially small superpotential in Sec. III. Section IV is devoted to conclusions and discussions. In the Appendix, we describe a detailed discussion about various perturbative vacuum solutions of our example.

\section{F-THEORY COMPACTIFICATIONS}

Here we describe several basic ingredients about spacetime compactifications in F-theory framework. For more details, we refer the reader to [8] and references therein. We also outline our strategy to find out a class of perturbatively flat vacua, which becomes a key ingredient to realize the small vacuum expectation value of the flux superpotential in a subsequent discussion.

\section{A. Basic setup}

Here we first take a brief look at general aspects of fourdimensional $\mathcal{N}=1$ effective theory arising from F-theory compactified on a Calabi-Yau fourfold $X_{4}$. In terms of the four-dimensional $\mathcal{N}=1$ supersymmetry, effective interactions of moduli fields can be determined by the Kähler potential and superpotential. Calabi-Yau compactifications enable us to derive these ingredients quantum mechanically from characteristics of the underlying Calabi-Yau manifolds.

More precisely, the Kähler potential for complex structure moduli space of $X_{4}$ is defined by

$$
K=-\ln \int_{X_{4}} \Omega \wedge \bar{\Omega},
$$

where $\Omega$ denotes a holomorphic $(4,0)$-form on $X_{4}$. Here and in what follows, we adopt the reduced Planck unit $M_{\mathrm{Pl}}=2.4 \times 10^{18} \mathrm{GeV}=1$. In the presence of background four-form fluxes $G_{4}$, Calabi-Yau compactifications also admit a flux superpotential of the form [9]

$$
W=\int_{X_{4}} G_{4} \wedge \Omega,
$$

which is inherited from a duality between the F-theory and M-theory compactified on the same manifold [10-13]. Here background fluxes are required to satisfy the tadpole cancellation condition given by

$$
\frac{\chi}{24}=n_{\mathrm{D} 3}+\frac{1}{2} \int_{X_{4}} G_{4} \wedge G_{4},
$$

in order to globally conserve the total charges within a compact manifold $X_{4}$. Here $\chi$ represents the Euler characteristic of $X_{4}$ and $n_{\mathrm{D} 3}$ denotes the total number of mobile D3-branes freely moving in $X_{4}$.

For a Calabi-Yau fourfold $X_{4}$ equipped with $h^{3,1}\left(X_{4}\right)$ complex structure moduli, the so-called period integrals of holomorphic $(4,0)$-form defined by

$$
\Pi_{A}=\int_{\gamma_{A}} \Omega
$$

encode the moduli dependence of the effective theory, and in particular the Kähler potential (2.1) for the complex structure moduli fields can be reexpressed as

$$
e^{-K}=\Pi \cdot \eta \cdot \bar{\Pi},
$$

where $\gamma_{A}$ with $A=1, \ldots, h_{H}^{4}\left(X_{4}\right)$ correspond to basis elements of primary horizontal subspace of $H_{4}\left(X_{4}\right)$. An intersection matrix $\eta_{A B}$ and a dual basis $\hat{\gamma}^{A}$ in $H_{H}^{4}\left(X_{4}\right)$ are defined accordingly by

$$
\eta^{A B}=\int_{X_{4}} \hat{\gamma}^{A} \wedge \hat{\gamma}^{B}, \quad \int_{\gamma_{A}} \hat{\gamma}^{B}=\delta_{A}^{B} .
$$

Similarly, if the underlying internal manifolds are filled with background four-form fluxes whose integer quantum numbers are given by

$$
n_{A}=\int_{\gamma_{A}} G_{4}
$$

moduli fields have interactions through the following form of the flux superpotential

$$
W=n_{A} \eta^{A B} \Pi_{B},
$$

in terms of period integrals.

\section{B. Our strategy}

Throughout this paper, we focus on a particular class of F-theory flux compactifications whose underlying Calabi-Yau fourfolds $X_{4}$ around a large complex structure point provide a specific type of moduli dependence into $W$ given by

$$
\begin{aligned}
W= & C_{0}\left(n_{A}\right)+\tilde{C}_{0}\left(n_{A}\right) S+C_{a}\left(n_{A}\right) z^{a}+\tilde{C}_{a}\left(n_{A}\right) S z^{a} \\
& +\frac{1}{2} C_{a b}\left(n_{A}\right) z^{a} z^{b}+\frac{1}{2} \tilde{C}_{a b}\left(n_{A}\right) S z^{a} z^{b} \\
& +\frac{1}{3 !} C_{a b c}\left(n_{A}\right) z^{a} z^{b} z^{c}+\frac{1}{3 !} \tilde{C}_{a b c}\left(n_{A}\right) S z^{a} z^{b} z^{c} \\
& +\frac{1}{4 !} C_{a b c d}\left(n_{A}\right) z^{a} z^{b} z^{c} z^{d}
\end{aligned}
$$


at the classical level. ${ }^{1}$ Here $a=1,2, \ldots, h^{3,1}\left(X_{4}\right)-1$ and the coefficients

$$
\left\{C_{0}, \tilde{C}_{0}, C_{a}, \tilde{C}_{a}, C_{a b}, \tilde{C}_{a b}, C_{a b c}, \tilde{C}_{a b c}, C_{a b c d}\right\}
$$

are functions of the background $G_{4}$-fluxes $n_{A}$, whose explicit form can be determined from topological data of $X_{4}$. Most important property of the above ansatz for F-theory superpotential is the existence of a particular moduli field $S$ which can enter only linearly in each terms of the polynomial. As we will show in the next section, we realize $S$ by a field originating from either the axio-dilaton or a linear combination of axio-dilaton and other moduli in the language of type IIB string theory.

Adopting the prescription developed in [4-6] into our present setup, it turns out that if there exists a restricted choice of $G_{4}$-fluxes whose nonzero contributions include primitive (2,2)-components (see for instance [8]) on a certain background such that the superpotential becomes a simple form ${ }^{2}$

$$
W=\tilde{C}_{a} S z^{a}+\frac{1}{2} C_{a b} z^{a} z^{b}
$$

a class of supersymmetric F-theory vacua satisfying

$$
\partial_{S} W=\partial_{a} W=W=0,
$$

can perturbatively exist along a one-dimensional locus

$$
z^{a}=S P^{a}
$$

if $P^{a} \equiv-\frac{1}{2}\left(C^{-1}\right)^{a b} \tilde{C}_{b}$ satisfy the following condition:

$$
\tilde{C}_{a} P^{a}=0 .
$$

Although stringy $\alpha^{\prime}$ corrections generically induce extra contributions proportional to $\zeta(3)$ into (2.9) at the perturbative level [14], the restricted choice of four-form fluxes picking up homogeneous of degree two terms does not support such a deformation, leading to the same result (2.11).

From a geometric point of view, the reduced form (2.11) can be realized from a certain combination of 3-point topological Yukawa couplings of the underlying CalabiYau fourfold explicitly given by

\footnotetext{
${ }^{1}$ In other words, here we assume that the background manifold has a certain fibration structure whose fiber has intersection number 0 with itself.

${ }^{2}$ In our approach, we started from just picking up homogeneous of degree two terms of the superpotential to realize the small $W$, which also simultaneously results in a discrete shift symmetric formula. Our prescription corresponds to provide a sufficient condition in the context of the original discussion in type IIB setup [4,5], and it would be interesting to clarify a necessary condition for the derivation along the lines of $[4,5]$.
}

$$
\frac{1}{2} n_{\alpha} \eta^{\alpha \beta} z^{i} z^{j} \int_{X_{4}} H_{\beta} \wedge J_{i} \wedge J_{j}
$$

where $\alpha$ labels the elements of primary subspace of the cohomology $H_{\text {prim }}^{2,2}\left(X_{4}\right) \subset H^{2,2}\left(X_{4}\right)$ whose bases $H_{\beta}$ are generated by the wedge products of the Kähler form $J_{i} \wedge J_{j}$. Correspondingly $n_{\alpha}$ represent (2,2)-components of the background $G_{4}$-flux quanta. Here we have assumed the existence of the appropriate modulus $S$ and utilized a unified expression for moduli space parameters as $z^{i} \equiv\left\{z^{a}, S\right\}$.

Once there exists an appropriate background admitting perturbative solutions of the above type, there would be a possibility for constructing desirable vacua where only the instanton corrections can contribute to the mass of the remaining modulus and induce a small superpotential, realizing a stable minima equipped with preferable properties. ${ }^{3}$

\section{AN EXPLICIT EXAMPLE}

In the remaining part of this paper, we focus on a specific elliptically-fibered Calabi-Yau fourfold to exemplify the realization of exponentially small vacuum expectation value of F-theory flux superpotential.

\section{A. Perturbatively flat vacua}

First we will show a detailed setup of our demonstration and elucidate the existence of the perturbatively flat solution of the above type, which becomes a key ingredient to realize desirable vacua after including nonperturbative corrections to the system.

Let us consider a mirror pair $\left(X_{4}, \tilde{X}_{4}\right)$ of Calabi-Yau fourfolds studied in [15], ${ }^{4}$ whose associated toric charge vectors $[18,19]$ are given $b^{5}$

$$
\begin{aligned}
& \ell^{1}=(0,-2,1,0,1,0,0,1,-1,0), \\
& \ell^{2}=(-6,1,0,0,0,2,3,0,0,0), \\
& \ell^{3}=(0,-1,0,1,0,0,0,-1,1,0), \\
& \ell^{4}=(0,-1,0,-1,0,0,0,1,0,1) .
\end{aligned}
$$

Their topological quantities such as the Hodge numbers and Euler characteristic are

\footnotetext{
${ }^{3}$ Since the identification of the modulus $S$ and determination of its appearance in flux superpotential strongly depends on the choice of manifolds and its triangulation, seeking a classification for suitable or unsuitable geometries for the mechanism would be quite difficult. However, there is no doubt that establishing a certain classification problem may provide a wide perspective about the subject.

${ }^{4}$ Here we would like to comment that we have also evaluated another simple Calabi-Yau fourfold studied in [16,17] and confirmed that there are no desirable perturbative solutions with flat directions, largely because in that case the tadpole cancellation condition becomes too severe.

${ }^{5}$ See $[15,20-23]$ for the details about explicit constructions of the background based on mirror symmetry techniques.
} 


$$
\begin{aligned}
h^{3,1}\left(X_{4}\right) & =h^{1,1}\left(\tilde{X}_{4}\right)=4, \\
h^{2,1}\left(X_{4}\right) & =h^{2,1}\left(\tilde{X}_{4}\right)=0, \\
h^{1,1}\left(X_{4}\right) & =h^{3,1}\left(\tilde{X}_{4}\right)=2796, \\
h^{2,2}\left(X_{4}\right) & =h^{2,2}\left(\tilde{X}_{4}\right)=11244, \\
\chi\left(X_{4}\right) & =\chi\left(\tilde{X}_{4}\right)=16848,
\end{aligned}
$$

and the dimension of primary horizontal subspace of $H_{4}\left(X_{4}\right)$ is given by $h_{H}^{4}\left(X_{4}\right)=16$, which also determines the total number of elements of the independent background fluxes $n_{A}$.

After a standard mirror symmetry calculation with the charge assignment in (3.1), one can show that there exist 16 independent period integrals associated with $X_{4}$ whose perturbative expansions are given by ${ }^{6}$

$$
\begin{aligned}
\Pi_{1}= & 1, \quad \Pi_{2}=z_{1}, \quad \Pi_{3}=z_{2}, \quad \Pi_{4}=z_{3}, \quad \Pi_{5}=z_{4}, \\
\Pi_{6}= & z_{2}\left(z_{2}+z_{3}+z_{4}\right), \quad \Pi_{7}=z_{2}\left(2 z_{1}+3 z_{2}+2 z_{3}\right), \quad \Pi_{8}=2 z_{2}\left(z_{1}+2 z_{2}+z_{3}+z_{4}\right), \\
\Pi_{9}= & 2 z_{1}^{2}+24 z_{1} z_{2}+\frac{103}{2} z_{2}^{2}+9 z_{1} z_{3}+34 z_{2} z_{3}+\frac{9}{2} z_{3}^{2}+7\left(z_{1}+3 z_{2}+z_{3}\right) z_{4}, \\
\Pi_{10}= & \frac{1}{2}\left[3 z_{1}^{2}+28 z_{1} z_{2}+55 z_{2}^{2}+10 z_{1} z_{3}+36 z_{2} z_{3}+5 z_{3}^{2}+6\left(z_{1}+3 z_{2}+z_{3}\right) z_{4}\right], \\
\Pi_{11}= & \frac{1}{2}\left[3 z_{1}^{2}+30 z_{1} z_{2}+61 z_{2}^{2}+10 z_{1} z_{3}+38 z_{2} z_{3}+5 z_{3}^{2}+8\left(z_{1}+3 z_{2}+z_{3}\right) z_{4}\right], \\
\Pi_{12}= & -\frac{z_{2}}{6}\left[11 z_{2}^{2}+6 z_{1}\left(z_{2}+z_{3}+z_{4}\right)+3 z_{3}\left(z_{3}+2 z_{4}\right)+3 z_{2}\left(4 z_{3}+3 z_{4}\right)\right]+\frac{165 i \zeta(3)}{2 \pi^{3}}, \\
\Pi_{13}= & -\frac{z_{2}}{6}\left(6 z_{1}^{2}+33 z_{1} z_{2}+46 z_{2}^{2}\right)+\frac{1}{2}\left(z_{1}+3 z_{2}\right)\left(z_{1}+5 z_{2}\right) z_{3}+\frac{1}{2}\left(z_{1}+4 z_{2}\right) z_{3}^{2}+\frac{z_{3}^{3}}{6} \\
& +\frac{z_{4}}{2}\left(z_{1}+3 z_{2}+z_{3}\right)^{2}+\frac{347 i \zeta(3)}{\pi^{3}}, \\
\Pi_{14}= & -\frac{z_{2}}{2}\left[z_{1}^{2}+5 z_{2}^{2}+4 z_{2} z_{3}+z_{3}^{2}+3 z_{2} z_{4}+2 z_{3} z_{4}+2 z_{1}\left(2 z_{2}+z_{3}+z_{4}\right)\right]+\frac{225 i \zeta(3)}{2 \pi^{3}}, \\
\Pi_{15}= & -\frac{z_{2}}{2}\left(z_{1}^{2}+3 z_{1} z_{2}+3 z_{2}^{2}+2 z_{1} z_{3}+3 z_{2} z_{3}+z_{3}^{2}\right)+\frac{135 i \zeta(3)}{2 \pi^{3}}, \\
\Pi_{16}= & \frac{z_{2}}{12}\left[22 z_{1} z_{2}^{2}+23 z_{2}^{3}+6 z_{1}^{2}\left(z_{2}+z_{3}+z_{4}\right)+6 z_{1} z_{3}\left(z_{3}+2 z_{4}\right)+2 z_{3}^{2}\left(z_{3}+3 z_{4}\right)+6 z_{2} z_{3}\left(2 z_{3}+3 z_{4}\right)\right. \\
& \left.+6 z_{1} z_{2}\left(4 z_{3}+3 z_{4}\right)+6 z_{2}^{2}\left(5 z_{3}+3 z_{4}\right)\right]-\frac{i\left(165 z_{1}+694 z_{2}+225 z_{3}+135 z_{4}\right) \zeta(3)}{2 \pi^{3}},
\end{aligned}
$$

around a large complex structure point $z_{1,2,3,4}=\infty$. Here we have abbreviated further possible corrections originating from world sheet instantons in the topological A-model, in order to restrict ourselves, at this stage, to the perturbative analysis.

In this fourfold example, the associated intersection matrix defined in (2.6) becomes

$$
\eta=\left(\begin{array}{ccccc}
0 & 0 & 0 & 0 & 1 \\
0 & 0 & 0 & I_{4} & 0 \\
0 & 0 & \tilde{\eta} & 0 & 0 \\
0 & I_{4} & 0 & 0 & 0 \\
1 & 0 & 0 & 0 & 0
\end{array}\right)
$$

\footnotetext{
${ }^{6}$ Note that our present notation for the complex structure moduli fields $\{z\}$ deviates from a standard convention where the classical periods are expressed by logarithmic functions of the complex structure deformations. We have redefined such a logarithm of a standard complex structure modulus as a new single modulus, just for the later convenience.
} 
with

$$
\tilde{\eta}=\left(\begin{array}{cccccc}
2 & 0 & -1 & -\frac{5}{7} & \frac{1}{7} & \frac{8}{7} \\
0 & \frac{1}{2} & -\frac{3}{4} & 0 & -\frac{1}{2} & \frac{1}{2} \\
-1 & -\frac{3}{4} & \frac{1}{2} & \frac{3}{14} & \frac{5}{14} & -\frac{9}{14} \\
-\frac{5}{7} & 0 & \frac{3}{14} & 0 & 0 & 0 \\
\frac{1}{7} & -\frac{1}{2} & \frac{5}{14} & 0 & 0 & 0 \\
\frac{8}{7} & \frac{1}{2} & -\frac{9}{14} & 0 & 0 & 0
\end{array}\right),
$$

and the explicit form of the flux superpotential is given by

$$
\begin{aligned}
W= & n_{16}+n_{15} z_{4}+n_{12} z_{1}+n_{13} z_{2}+n_{14} z_{3}+\frac{z_{4}}{14}\left(7 n_{7} z_{1}+12 n_{10} z_{2}-2 n_{11} z_{2}-4 n_{9} z_{2}+7 n_{7} z_{3}\right) \\
& +\frac{1}{28}\left(14 n_{6} z_{1}^{2}-8 n_{10} z_{1} z_{2}-8 n_{11} z_{1} z_{2}+12 n_{9} z_{1} z_{2}+2 n_{10} z_{2}^{2}+2 n_{11} z_{2}^{2}+4 n_{9} z_{2}^{2}+14 n_{8} z_{1} z_{3}\right. \\
& \left.-4 n_{10} z_{2} z_{3}+24 n_{11} z_{2} z_{3}-8 n_{9} z_{2} z_{3}+7 n_{8} z_{3}^{2}\right)-\frac{z_{4}}{2}\left(n_{3} z_{1}^{2}+2 n_{2} z_{1} z_{2}+6 n_{3} z_{1} z_{2}+2 n_{4} z_{1} z_{2}\right. \\
& \left.+3 n_{2} z_{2}^{2}+9 n_{3} z_{2}^{2}+3 n_{4} z_{2}^{2}+2 n_{3} z_{1} z_{3}+2 n_{2} z_{2} z_{3}+6 n_{3} z_{2} z_{3}+2 n_{4} z_{2} z_{3}+n_{3} z_{3}^{2}\right) \\
& -\frac{1}{6}\left(3 n_{4} z_{1}^{2} z_{2}+6 n_{3} z_{1}^{2} z_{2}+3 n_{5} z_{1}^{2} z_{2}+6 n_{2} z_{1} z_{2}^{2}+33 n_{3} z_{1} z_{2}^{2}+12 n_{4} z_{1} z_{2}^{2}+9 n_{5} z_{1} z_{2}^{2}+11 n_{2} z_{2}^{3}\right. \\
& +46 n_{3} z_{2}^{3}+15 n_{4} z_{2}^{3}+9 n_{5} z_{2}^{3}+3 n_{3} z_{1}^{2} z_{3}+6 n_{2} z_{1} z_{2} z_{3}+24 n_{3} z_{1} z_{2} z_{3}+6 n_{4} z_{1} z_{2} z_{3}+6 n_{5} z_{1} z_{2} z_{3} \\
& +12 n_{2} z_{2}^{2} z_{3}+45 n_{3} z_{2}^{2} z_{3}+12 n_{4} z_{2}^{2} z_{3}+9 n_{5} z_{2}^{2} z_{3}+3 n_{3} z_{1} z_{3}^{2}+3 n_{2} z_{2} z_{3}^{2}+12 n_{3} z_{2} z_{3}^{2}+3 n_{4} z_{2} z_{3}^{2} \\
& \left.+3 n_{5} z_{2} z_{3}^{2}+n_{3} z_{3}^{3}\right)+\frac{z_{4}}{2}\left(n_{1} z_{1}^{2} z_{2}+3 n_{1} z_{1} z_{2}^{2}+3 n_{1} z_{2}^{3}+2 n_{1} z_{1} z_{2} z_{3}+3 n_{1} z_{2}^{2} z_{3}+n_{1} z_{2} z_{3}^{2}\right) \\
& +\frac{1}{12}\left(6 n_{1} z_{1}^{2} z_{2}^{2}+22 n_{1} z_{1} z_{2}^{3}+23 n_{1} z_{2}^{4}+6 n_{1} z_{1}^{2} z_{2} z_{3}+24 n_{1} z_{1} z_{2}^{2} z_{3}+30 n_{1} z_{2}^{3} z_{3}+6 n_{1} z_{1} z_{2} z_{3}^{2}\right. \\
& \left.+12 n_{1} z_{2}^{2} z_{3}^{2}+2 n_{1} z_{2} z_{3}^{3}\right)+\frac{i \zeta(3)}{2 \pi^{3}}\left[165 n_{2}+694 n_{3}+225 n_{4}+135 n_{5}-n_{1}\left(165 z_{1}+694 z_{2}\right.\right. \\
& \left.\left.+225 z_{3}+135 z_{4}\right)\right],
\end{aligned}
$$

which enables us to identify the modulus $z_{4}$ inherited from the axio-dilaton as $S$ explained in Sec. II B. Note that we rewrite $z_{4}$ as $S$ in subsequent discussions. At the perturbative level, the dynamics of effective theory arising from F-theory compactified on $X_{4}$ can be completely specified by (3.6) and the Kähler potential (2.5) explicitly given by

$$
\begin{aligned}
K= & (S-\bar{S})\left[\frac{\left(z_{1}-\bar{z}_{1}\right)^{2}\left(z_{2}-\bar{z}_{2}\right)}{2}+\frac{3\left(z_{1}-\bar{z}_{1}\right)\left(z_{2}-\bar{z}_{2}\right)^{2}}{2}+\frac{3\left(z_{2}-\bar{z}_{2}\right)^{3}}{2}+\left(z_{1}-\bar{z}_{1}\right)\left(z_{2}-\bar{z}_{2}\right)\left(z_{3}-\bar{z}_{3}\right)\right. \\
& \left.+\frac{3\left(z_{2}-\bar{z}_{2}\right)^{2}\left(z_{3}-\bar{z}_{3}\right)}{2}+\frac{\left(z_{2}-\bar{z}_{2}\right)\left(z_{3}-\bar{z}_{3}\right)^{2}}{2}\right]+\frac{\left(z_{1}-\bar{z}_{1}\right)^{2}\left(z_{2}-\bar{z}_{2}\right)^{2}}{2}+\frac{11\left(z_{1}-\bar{z}_{1}\right)\left(z_{2}-\bar{z}_{2}\right)^{3}}{6} \\
& +\frac{23\left(z_{2}-\bar{z}_{2}\right)^{4}}{12}+\frac{\left(z_{1}-\bar{z}_{1}\right)^{2}\left(z_{2}-\bar{z}_{2}\right)\left(z_{3}-\bar{z}_{3}\right)}{2}+2\left(z_{1}-\bar{z}_{1}\right)\left(z_{2}-\bar{z}_{2}\right)^{2}\left(z_{3}-\bar{z}_{3}\right) \\
& +\frac{5\left(z_{2}-\bar{z}_{2}\right)^{3}\left(z_{3}-\bar{z}_{3}\right)}{2}+\frac{\left(z_{1}-\bar{z}_{1}\right)\left(z_{2}-\bar{z}_{2}\right)\left(z_{3}-\bar{z}_{3}\right)^{2}}{2}+\left(z_{2}-\bar{z}_{2}\right)^{2}\left(z_{3}-\bar{z}_{3}\right)^{2} \\
& +\frac{\left(z_{2}-\bar{z}_{2}\right)\left(z_{3}-\bar{z}_{3}\right)^{3}}{6}+\frac{i \zeta(3)}{4 \pi^{3}}\left[-660\left(z_{1}-\bar{z}_{1}\right)-540(S-\bar{S})-900\left(z_{3}-\bar{z}_{3}\right)-2776\left(z_{2}-\bar{z}_{2}\right)\right]
\end{aligned}
$$

Now let us check whether the above example of F-theory flux compactifications appropriately admits a class of perturbative supersymmetric solution with a flat direction discussed in the previous section. When the background fourform $G_{4}$-fluxes are restricted to be of the type $(2,2)$ and primitive as represented by setting 


$$
n_{1}=n_{2}=n_{3}=n_{4}=n_{5}=n_{12}=n_{13}=n_{14}=n_{15}=n_{16}=0,
$$

one can easily show that the flux superpotential of the model becomes a desirable form (2.11) with the following assignments:

$$
\tilde{C}_{1}=\frac{n_{7}}{2}, \quad \tilde{C}_{2}=\frac{1}{7}\left(6 n_{10}-n_{11}-2 n_{9}\right), \quad \tilde{C}_{3}=\frac{n_{7}}{2},
$$

and

$$
\left(\begin{array}{lll}
C_{11} & C_{12} & C_{13} \\
C_{21} & C_{22} & C_{23} \\
C_{31} & C_{32} & C_{33}
\end{array}\right)=\left(\begin{array}{ccc}
n_{6} & \frac{-2 n_{10}-2 n_{11}+3 n_{9}}{7} & \frac{n_{8}}{2} \\
\frac{-2 n_{10}-2 n_{11}+3 n_{9}}{7} & \frac{n_{10}+n_{11}+2 n_{9}}{7} & -\frac{n_{10}-6 n_{11}+2 n_{9}}{7} \\
\frac{n_{8}}{2} & -\frac{n_{10}-6 n_{11}+2 n_{9}}{7} & \frac{n_{8}}{2}
\end{array}\right) .
$$

Note that the nonzero (2,2)-components of the background fluxes must be chosen such that the matrix $C_{a b}$ in (3.10) continues to be invertible and the condition (2.14) with the assignments

$$
\begin{aligned}
P^{1}= & \frac{1}{\mathcal{A}}\left(n_{10}+8 n_{11}+5 n_{9}\right)\left(-n_{11}\left(6 n_{7}+n_{8}\right)+n_{10}\left(n_{7}+6 n_{8}\right)+2\left(n_{7}-n_{8}\right) n_{9}\right) \\
P^{2}= & \frac{7}{2 \mathcal{A}}\left(2 n_{6}-n_{8}\right)\left(-n_{11}\left(6 n_{7}+n_{8}\right)+n_{10}\left(n_{7}+6 n_{8}\right)+2\left(n_{7}-n_{8}\right) n_{9}\right) \\
P^{3}= & \frac{2 n_{10}^{2}}{\mathcal{A}}\left(6 n_{6}-n_{7}-6 n_{8}\right)+\frac{2 n_{11}^{2}}{\mathcal{A}}\left(6 n_{6}-8 n_{7}+n_{8}\right)+\frac{n_{11}}{2 \mathcal{A}}\left(14 n_{6} n_{7}-7 n_{7} n_{8}+40 n_{6} n_{9}+68 n_{7} n_{9}+2 n_{8} n_{9}\right) \\
& -\frac{n_{9}}{\mathcal{A}}\left(-14 n_{6} n_{7}+7 n_{7} n_{8}+8 n_{6} n_{9}+15 n_{7} n_{9}+6 n_{8} n_{9}\right) \\
& +\frac{n_{10}}{\mathcal{A}}\left(14 n_{6} n_{7}-7 n_{7} n_{8}-4 n_{11}\left(37 n_{6}+9 n_{7}+5 n_{8}\right)+40 n_{6} n_{9}+26 n_{7} n_{9}+44 n_{8} n_{9}\right) \\
\mathcal{A} \equiv & 4 n_{10}^{2} n_{6}+8 n_{11}^{2}\left(18 n_{6}+7 n_{8}\right)+7 n_{11} n_{8}\left(n_{8}-16 n_{9}\right)-2 n_{11} n_{6}\left(7 n_{8}+48 n_{9}\right)+2 n_{9}\left(7 n_{8}\left(n_{8}+3 n_{9}\right)+n_{6}\left(-14 n_{8}+8 n_{9}\right)\right) \\
& +n_{10}\left(8 n_{11}\left(-6 n_{6}+7 n_{8}\right)+7 n_{8}\left(n_{8}-4 n_{9}\right)+2 n_{6}\left(-7 n_{8}+8 n_{9}\right)\right)
\end{aligned}
$$

are also satisfied. ${ }^{7}$

Here let us pick up a particular choice of background fluxes whose nonzero components are

$$
\begin{aligned}
& n_{6}=-10, \quad n_{7}=n_{11}=-8, \quad n_{8}=12, \\
& n_{9}=7, \quad n_{10}=15 .
\end{aligned}
$$

In order to satisfy the tadpole cancellation condition (2.3) of the present model, the background also needs to contain additional $n_{D 3}=2$ mobile D3-branes, whose numbers are sufficiently small to guarantee our exclusion of the backreaction to the space-time. After plugging these inputs into the general formula (2.13), one finds that the model admits

\footnotetext{
${ }^{7}$ Here we have omitted the possibility of another type of perturbatively flat solution satisfying $\operatorname{Det}\left(C_{a b}\right)=0$ and more detailed analysis of the vacuum solutions of the present example are relegated to the Appendix.
}

a desirable minimum equipped with a perturbative flat direction along

$$
\left(\begin{array}{l}
z_{1} \\
z_{2} \\
z_{3}
\end{array}\right)=\frac{S}{7}\left(\begin{array}{l}
3 \\
4 \\
9
\end{array}\right) .
$$

This solution corresponds to the "vacuum A" specified in the next section.

\section{B. Nonperturbative uplifting of flat direction}

By using algebraic methods of the toric geometry (see for example [24,25]), one can determine nonperturbative quantum corrections in the moduli space of Calabi-Yau manifolds by solving the associated Picard-Fuchs equations and calculating the period integrals explicitly. For the fourfold example described in the previous subsection, it turns out that the corresponding period integrals (3.3) take the following form at the nonperturbative level: 


$$
\begin{aligned}
\tilde{\Pi}_{1}= & \Pi_{1}=1, \quad \tilde{\Pi}_{2}=\Pi_{2}=z_{1}, \quad \tilde{\Pi}_{3}=\Pi_{3}=z_{2}, \quad \tilde{\Pi}_{4}=\Pi_{4}=z_{3}, \quad \tilde{\Pi}_{5}=\Pi_{5}=S, \\
\tilde{\Pi}_{6}= & \Pi_{6}+\frac{e^{2 \pi i z_{1}}}{4 \pi^{2}}, \quad \tilde{\Pi}_{7}=\Pi_{7}-\frac{e^{2 \pi i S}}{2 \pi^{2}}, \quad \tilde{\Pi}_{8}=\Pi_{8}-\frac{e^{2 \pi i z_{3}}}{2 \pi^{2}}, \quad \tilde{\Pi}_{9}=\Pi_{9}-\frac{1545 e^{2 \pi i z_{2}}}{\pi^{2}}, \\
\tilde{\Pi}_{10}= & \Pi_{10}-\frac{825 e^{2 \pi i z_{2}}}{\pi^{2}}, \quad \tilde{\Pi}_{11}=\Pi_{11}-\frac{915 e^{2 \pi i z_{2}}}{\pi^{2}}, \\
\tilde{\Pi}_{12}= & \Pi_{12}+\frac{1}{8 \pi^{3}}\left[e^{2 \pi i z_{1}}\left(i+2 \pi z_{1}\right)-1320 e^{2 \pi i z_{2}}\left(i+\pi z_{2}\right)-2 e^{2 \pi i z_{3}}\left(i+\pi z_{3}\right)-2 e^{2 \pi i S}(i+\pi S)\right], \\
\tilde{\Pi}_{13}= & \Pi_{13}-\frac{15 e^{2 \pi i z_{2}}}{\pi^{3}}\left[23 i+\pi\left(11 z_{1}+46 z_{2}+15 z_{3}+9 S\right)\right], \\
\tilde{\Pi}_{14}= & \Pi_{14}-\frac{1}{8 \pi^{3}}\left[1800 e^{2 \pi i z_{2}}\left(i+\pi z_{2}\right)+e^{2 \pi i z_{3}}\left(i+2 \pi\left(z_{1}+z_{3}\right)\right)+2 e^{2 \pi i S}(i+\pi S)\right], \\
\tilde{\Pi}_{15}= & \Pi_{15}-\frac{1}{4 \pi^{3}}\left[540 e^{2 \pi i z_{2}}\left(i+\pi z_{2}\right)+e^{2 \pi i S} \pi\left(z_{1}+z_{3}\right)\right], \\
\tilde{\Pi}_{16}= & \Pi_{16}-\frac{1}{8 \pi^{3}}\left[-e^{2 \pi i z_{1}} z_{1}\left(i+\pi z_{1}\right)+e^{2 \pi i z_{3}}\left(2 z_{1}+z_{3}\right)\left(i+\pi z_{3}\right)+2 e^{2 \pi i S}\left(z_{1}+z_{3}\right)(i+\pi S)\right. \\
& \left.+120 e^{2 \pi i z_{2}}\left(i+\pi z_{2}\right)\left(11 z_{1}+23 z_{2}+15 z_{3}+9 S\right)\right] .
\end{aligned}
$$

Here $\Pi_{i}$ just denote the perturbative expansions of period integrals described in (3.3) and we have added leading corrections due to the one-instanton $\mathcal{O}\left(e^{-2 \pi \operatorname{Im}(z)}\right)$ effect from the A-model perspective, which is sufficient to exemplify the uplifting mechanism realizing F-theory vacua with small superpotential.

Accordingly, the Kähler potential (3.7) is also deformed into the following expression:

$$
\begin{aligned}
\tilde{K}= & K-\frac{\operatorname{Im}\left(z_{1}\right)\left(1+2 \pi \operatorname{Im}\left(z_{1}\right)\right)}{2 \pi^{3}} e^{-2 \pi \operatorname{Im}\left(z_{1}\right)} \cos \left(2 \pi \operatorname{Re}\left(z_{1}\right)\right) \\
& +\frac{120\left(1+2 \pi \operatorname{Im}\left(z_{2}\right)\right)\left(11 \operatorname{Im}\left(z_{1}\right)+23 \operatorname{Im}\left(z_{2}\right)+15 \operatorname{Im}\left(z_{3}\right)+9 \operatorname{Im}(S)\right)}{2 \pi^{3}} e^{-2 \pi \operatorname{Im}\left(z_{2}\right)} \cos \left(2 \pi \operatorname{Re}\left(z_{2}\right)\right) \\
& +\frac{\left(1+2 \pi \operatorname{Im}\left(z_{3}\right)\right)\left(2 \operatorname{Im}\left(z_{1}\right)+\operatorname{Im}\left(z_{3}\right)\right)}{2 \pi^{3}} e^{-2 \pi \operatorname{Im}\left(z_{3}\right)} \cos \left(2 \pi \operatorname{Re}\left(z_{3}\right)\right) \\
& +\frac{2(1+2 \pi \operatorname{Im}(S))\left(\operatorname{Im}\left(z_{1}\right)+\operatorname{Im}\left(z_{3}\right)\right)}{2 \pi^{3}} e^{-2 \pi \operatorname{Im}(S)} \cos (2 \pi \operatorname{Re}(S)) .
\end{aligned}
$$

As mentioned before, we rely on a numerical calculation to find out desirable F-theory flux vacua with exponentially small superpotential. More precisely, in order to demonstrate the nonperturbative uplifting mechanism, we utilized the Mathematica to solve the intricate differential system (2.12), and analyzed the vacuum structure of the present model under the following range of background fluxes:

$$
-20 \leq n_{6}, n_{7}, n_{8}, n_{9}, n_{10}, n_{11} \leq 20 .
$$

The tadpole cancellation condition (2.3) whose explicit form is given by

$$
\begin{aligned}
702= & n_{D 3}+n_{1} n_{16}+n_{2} n_{12}+n_{3} n_{13}+n_{4} n_{14}+n_{5} n_{15} \\
& +\frac{1}{7} n_{6} n_{10}+\frac{8}{7} n_{6} n_{11}+n_{6}^{2}-\frac{1}{2} n_{7} n_{10} \\
& +\frac{1}{2} n_{7} n_{11}+\frac{1}{4} n_{7}^{2}+\frac{5}{14} n_{8} n_{10}-\frac{9}{14} n_{8} n_{11}-n_{6} n_{8} \\
& -\frac{3}{4} n_{7} n_{8}+\frac{1}{4} n_{8}^{2}-\frac{5}{7} n_{6} n_{9}+\frac{3}{14} n_{8} n_{9}
\end{aligned}
$$

must be also satisfied. Note that we have also restricted ourselves to a small number of mobile D3-branes as $0 \leq n_{D 3} \leq 10$, in order to legitimately exclude the effect of backreaction to the space-time.

As a result, we found that there are 18 perturbatively flat solutions of the type given by (2.13) in the present setup, 
TABLE I. Explicit values of the stabilized moduli fields and small flux superpotential in $M_{\mathrm{Pl}}=1$ unit.

\begin{tabular}{lccccc}
\hline \hline Vacuum & $z_{1}$ & $z_{2}$ & $z_{3}$ & $S$ & $\left|W_{0}\right|$ \\
\hline $\mathrm{A}$ & $1.95 i$ & $2.60 i$ & $5.86 i$ & $4.56 i$ & $6.75 \times 10^{-9}$ \\
$\mathrm{~B}$ & $1.35 i$ & $1.97 i$ & $4.06 i$ & $4.06 i$ & $6.11 \times 10^{-7}$ \\
$\mathrm{C}$ & $2.41 i$ & $1.81 i$ & $1.20 i$ & $2.71 i$ & $2.50 \times 10^{-6}$ \\
\hline \hline
\end{tabular}

TABLE II. Mass squareds of canonically normalized moduli fields in $M_{\mathrm{Pl}}=1$ unit.

\begin{tabular}{lc}
\hline \hline Vacuum & Eigenvalues of mass matrix $\partial_{I} \partial_{J} V \times \mathcal{V}^{2}$ \\
\hline A & $(24.7,24.7,4.86,4.86,0.634,0.634$, \\
& $\left.9.79 \times 10^{-14}, 9.65 \times 10^{-14}\right)$ \\
B & $(42.5,42.5,8.76,8.76,1.33,1.33$, \\
& $\left.4.68 \times 10^{-10}, 4.56 \times 10^{-10}\right)$ \\
C & $(61.9,61.9,15.2,15.2,0.765,0.765$, \\
& $\left.1.30 \times 10^{-8}, 1.27 \times 10^{-8}\right)$ \\
\hline \hline
\end{tabular}

and after including the instanton corrections to the potential, 3 out of them remain to satisfy the differential system (2.12) and realize desirable class of flux vacua with small superpotential. ${ }^{8}$ Here we denote these 3 different solutions as vacua $\mathrm{A}, \mathrm{B}$, and $\mathrm{C}$, whose background data such as the $G_{4}$-flux quanta and number of mobile D3-branes have been determined as follows:

\begin{tabular}{lcc}
\hline Vacuum & Set of fluxes $\left(n_{6}, n_{7}, n_{8}, n_{9}, n_{10}, n_{11}\right)$ & $n_{D 3}$ \\
\hline $\mathrm{A}$ & $(-10,-8,12,7,15,-8)$ & 2 \\
$\mathrm{~B}$ & $(-9,-8,14,0,11,-11)$ & 5 \\
$\mathrm{C}$ & $(-15,8,6,20,-4,-8)$ & 6 \\
\hline
\end{tabular}

The explicit values of the superpotential $W_{0} \equiv\left\langle e^{K / 2} W\right\rangle$, stabilized moduli fields and their mass squareds at each of the flux vacua are summarized in Tables I and II, where $V$ and $\mathcal{V}$ are the scalar potential and overall volume of the background respectively. In the case of vacuum A, the perturbatively flat direction along (3.13) has been stabilized due to a small nonperturbative contribution to the superpotential of a racetrack-type given by

$W=\frac{1}{4 \pi^{2}}\left[-44 e^{\frac{6 \pi i S}{7}}+2040 e^{\frac{8 \pi i S}{7}}+49 e^{2 \pi i S}-68 e^{\frac{18 \pi i S}{7}}\right]$,

and the perturbative shift symmetry is broken down to a discrete subgroup. Here we used the fact that orthogonal directions to the flat direction are all heavy and can be integrated out. Note that light modes originating from the uplifted flat direction can be involved with the dynamics of Kähler moduli fields, as discussed in [4-6].

\footnotetext{
${ }^{8}$ To guarantee the convergence of the expansion around a large complex structure point of the period integrals and the superpotential, we picked up numerical solutions satisfying the conditions $\operatorname{Im}\left(z_{i}\right)>1$ only.
}

It is worth noting that one can straightforwardly generalize our analysis to include higher-order instanton corrections as well. Especially, we confirmed that the above numerical solutions with tiny flux superpotential continue to be stable against the next instanton corrections.

\section{CONCLUSIONS AND DISCUSSIONS}

Toward an explicit realization of our four-dimensional physics within the framework of the general concept of F-theory, we explored the possibility of the realization of F-theory flux compactifications with exponentially small superpotential. It has been recently pointed out in $[4,5]$ that nonperturbative corrections to complex structure moduli fields can naturally give rise to tiny flux superpotential in type IIB string theory on Calabi-Yau threefolds. Since the smallness of the flux superpotential plays a crucial role in KKLT-type construction for de Sitter space, we examined whether such a simple but broadly applicable method can be also realized in F-theory compactified on Calabi-Yau fourfolds.

Generalizing the type IIB setups analyzed in $[4,5]$ into the frameworks of F-theory compactifications, we clarified that a restricted choice of $G_{4}$-flux components reduces the flux superpotential into a quite simple form of homogeneous of degree two, and a class of supersymmetric F-theory vacua can perturbatively exist along with one flat direction. Then we determined one-instanton corrections to the potential of a particular example by utilizing the techniques of mirror symmetry, and numerically investigated its vacuum structure at the nonperturbative level.

As a result, we numerically confirmed that flat directions of perturbative vacua of the model can be lifted appropriately and remaining modulus acquired a small mass, along with a desired tiny superpotential. Although our explicit demonstration of this uplifting mechanism has the potential for tremendous impact on the implementation of KKLT construction in a broad range of F-theory frameworks, rigorous calculation about Kähler moduli stabilization in F-theory requires more precise understanding about strong dynamics of seven-branes, remaining an open problem.

From a statistical point of view, it would be interesting to clarify to what extent flux vacua with small superpotential distribute inside the string/F-theory landscape. One possible approach to address this intriguing subject is to promote discrete $G_{4}$ fluxes to continuous parameters and attempt to obtain a reasonable estimate for the numbers of possible F-theory vacua, as initiated in [26-28] for type IIB flux compactifications.

Moreover, the authors of $[5,6]$ recently applied the nonperturbative uplifting mechanism to the conifold region of Calabi-Yau moduli space and explicitly found conifold vacua with small flux superpotential. Although a 
comprehensive study about global structure of moduli space of Calabi-Yau fourfolds has not yet been fully elucidated, it is also fascinating to extend our F-theory setup into other corners of the landscape in the future.

\section{ACKNOWLEDGMENTS}

We would like to thank K. Ohta for useful discussions and comments, and especially to the anonymous referee for a careful reading of the manuscript and constructive suggestions. H. O. was supported in part by JSPS KAKENHI Grants No. JP19J00664 and No. JP20K14477.

\section{APPENDIX: ANOTHER TYPE OF PERTURBATIVE SOLUTION}

Here we comment on the existence of another type of perturbatively flat vacua purposefully omitted from the main text. Although the matrix $C_{a b}$ has been assumed to be invertible throughout this paper, generically it is also possible to realize desirable solutions equipped with flat directions, even when $\operatorname{Det}\left(C_{a b}\right)=0$. In the case of the example described in Sec. III A, one can easily show that there exist two more perturbative solutions depending on the choice of $G_{4}$-fluxes as follows:

(i) Under the condition

$$
\begin{aligned}
& n_{7}=0, \\
& n_{8}=-3 n_{10}+4 n_{11}+n_{6}+\frac{-5 n_{11}^{2}+\sqrt{\left(\left(6 n_{10}-5 n_{11}\right)^{2}-4 n_{10} n_{6}\right)\left(\left(2 n_{10}-n_{11}\right)^{2}-4 n_{10} n_{6}\right)}}{4 n_{10}}, \\
& n_{9}=3 n_{10}-\frac{n_{11}}{2},
\end{aligned}
$$

there exists a solution to (2.12) satisfying $\operatorname{Det}\left(C_{a b}\right)=\tilde{C}_{a}=0$ with an additional flat direction given by

$$
\left(\begin{array}{c}
z_{2} \\
z_{3}
\end{array}\right)=z_{1}\left(\begin{array}{c}
\frac{-12 n_{10}^{2}+16 n_{10} n_{11}-5 n_{11}^{2}-4 n_{10} n_{6}+\sqrt{\left(\left(6 n_{10}-5 n_{11}\right)^{2}-4 n_{10} n_{6}\right)\left(\left(-2 n_{10}+n_{11}\right)^{2}-4 n_{10} n_{6}\right)}}{4 n_{10}\left(4 n_{10}-3 n_{11}\right)} \\
\frac{\left(-2 n_{10}+n_{11}\right)^{2}-4 n_{10} n_{6}+\sqrt{\left(\left(6 n_{10}-5 n_{11}\right)^{2}-4 n_{10} n_{6}\right)\left(\left(-2 n_{10}+n_{11}\right)^{2}-4 n_{10} n_{6}\right)}}{4\left(4 n_{10}-3 n_{11}\right)\left(n_{10}-n_{11}\right)}
\end{array}\right),
$$

as well as the obvious flat direction parametrized by $S$.

(ii) Under the condition

$$
\begin{aligned}
& n_{6}=-\frac{n_{7}\left(16 n_{10}^{2}+n_{10}\left(-44 n_{11}+5 n_{7}\right)+3 n_{11}\left(8 n_{11}+5 n_{7}\right)\right)}{4\left(4 n_{10}-3 n_{11}\right)^{2}}, \\
& n_{8}=2 n_{6}, \\
& n_{9}=\frac{n_{10}+8 n_{11}}{5},
\end{aligned}
$$

there exists a solution to (2.12) satisfying $\operatorname{Det}\left(C_{a b}\right)=0$ with a flat direction given by

$$
\left(\begin{array}{c}
z_{2} \\
S
\end{array}\right)=\left(z_{1}+z_{3}\right)\left(\begin{array}{c}
-\frac{5 n_{7}}{8 n_{10}-6 n_{11}} \\
\frac{8 n_{10}^{2}+n_{10}\left(-22 n_{11}+5 n_{7}\right)+3 n_{11}\left(4 n_{11}+5 n_{7}\right)}{2\left(4 n_{10}-3 n_{11}\right)^{2}}
\end{array}\right),
$$

as well as a direction given by $z_{1}-z_{3}$ locus.

The nonperturbative uplifting mechanism explicitly demonstrated in Sec. III B is presumed to be straightforwardly applied to this type of solutions. 
[1] S. B. Giddings, S. Kachru, and J. Polchinski, Hierarchies from fluxes in string compactifications, Phys. Rev. D 66 (2002) 106006.

[2] S. Kachru, R. Kallosh, A. D. Linde, and S. P. Trivedi, de Sitter vacua in string theory, Phys. Rev. D 68 (2003) 046005.

[3] V. Balasubramanian, P. Berglund, J. P. Conlon, and F. Quevedo, Systematics of moduli stabilisation in CalabiYau flux compactifications, J. High Energy Phys. 03 (2005) 007.

[4] M. Demirtas, M. Kim, L. Mcallister, and J. Moritz, Vacua with Small Flux Superpotential, Phys. Rev. Lett. 124, 211603 (2020).

[5] M. Demirtas, M. Kim, L. Mcallister, and J. Moritz, Conifold Vacua with small flux superpotential, arXiv:2009.03312.

[6] R. Álvarez-García, R. Blumenhagen, M. Brinkmann, and L. Schlechter, Small flux superpotentials for type IIB flux vacua close to a conifold, arXiv:2009.03325.

[7] C. Vafa, Evidence for F theory, Nucl. Phys. B469, 403 (1996).

[8] F. Denef, Les Houches lectures on constructing string Vacua, Les Houches 87, 483 (2008).

[9] S. Gukov, C. Vafa, and E. Witten, Nucl. Phys. B584, 69 (2000); Erratum, Nucl. Phys. B608, 477 (2001).

[10] K. Becker and M. Becker, M theory on eight manifolds, Nucl. Phys. B477, 155 (1996).

[11] S. Sethi, C. Vafa, and E. Witten, Constraints on low dimensional string compactifications, Nucl. Phys. B480, 213 (1996).

[12] K. Dasgupta, G. Rajesh, and S. Sethi, M theory, orientifolds and G-flux, J. High Energy Phys. 08 (1999) 023.

[13] M. Haack and J. Louis, M theory compactified on CalabiYau fourfolds with background flux, Phys. Lett. B 507, 296 (2001).

[14] Y. Honma and M. Manabe, Exact Kahler potential for Calabi-Yau fourfolds, J. High Energy Phys. 05 (2013) 102.
[15] T. W. Grimm, A. Klemm, and D. Klevers, Five-brane superpotentials, blow-up geometries and SU(3) structure manifolds, J. High Energy Phys. 05 (2011) 113.

[16] Y. Honma and H. Otsuka, On the flux vacua in F-theory compactifications, Phys. Lett. B 774, 225 (2017).

[17] Y. Honma and H. Otsuka, F-theory flux Vacua and attractor equations, J. High Energy Phys. 04 (2020) 001.

[18] V. V. Batyrev, Dual polyhedra and mirror symmetry for Calabi-Yau hypersurfaces in toric varieties, arXiv:alg-geom/ 9310003.

[19] V. V. Batyrev and L. A. Borisov, On Calabi-Yau complete intersections in toric varieties, arXiv:alg-geom/9412017.

[20] M. Alim, M. Hecht, H. Jockers, P. Mayr, A. Mertens, and M. Soroush, Hints for off-shell mirror symmetry in type II/ F-theory compactifications, Nucl. Phys. B841, 303 (2010).

[21] T. W. Grimm, T. W. Ha, A. Klemm, and D. Klevers, Computing brane and flux superpotentials in F-theory compactifications, J. High Energy Phys. 04 (2010) 015.

[22] H. Jockers, P. Mayr, and J. Walcher, On $N=14$ d effective couplings for F-theory and heterotic Vacua, Adv. Theor. Math. Phys. 14, 1433 (2010).

[23] Y. Honma and M. Manabe, Open mirror symmetry for higher dimensional Calabi-Yau Hypersurfaces, J. High Energy Phys. 03 (2016) 160.

[24] S. Hosono, A. Klemm, S. Theisen, and S. T. Yau, Mirror symmetry, mirror map and applications to Calabi-Yau hypersurfaces, Commun. Math. Phys. 167, 301 (1995).

[25] S. Hosono, A. Klemm, S. Theisen, and S. T. Yau, Mirror symmetry, mirror map and applications to complete intersection Calabi-Yau spaces, AMS/IP Stud. Adv. Math. 1, 545 (1996).

[26] S. Ashok and M. R. Douglas, Counting flux vacua, J. High Energy Phys. 01 (2004) 060.

[27] M. R. Douglas, The statistics of string/M theory vacua, J. High Energy Phys. 05 (2003) 046.

[28] F. Denef and M. R. Douglas, Distributions of flux vacua, J. High Energy Phys. 05 (2004) 072. 